\title{
COMPARATIVE STUDY OF THE RED AND VIOLET SYSTEMS OF CYANOGEN BANDS
}

\author{
Arthur S. King and P. Swings \\ Mount Wilson Observatory \\ Received September 23, 1944
}

\begin{abstract}
Comparison of bands belonging to the violet and red systems of $C N$ showed that in emission the $(1,0)$ band $\lambda 7873$ (red system) is stronger than the $(0,0)$ band $\lambda 3883$ (violet system) at temperatures of the electric furnace below $2300^{\circ} \mathrm{C}$. Approximate equality of the main structure of the two bands is attained at $2300^{\circ} \mathrm{C}$; $\lambda 3883$ becomes somewhat stronger than $\lambda 7873$ at $2600^{\circ} \mathrm{C}$. At the high temperature, approximately $7300^{\circ} \mathrm{C}$, of the carbon arc in air, the $\lambda 3883$ band is about two hundred times stronger than the $\lambda 7873$ band. Self-reversal appears easily in the violet band, but not in the red. The two systems react differently to a change in pressure, the red system increasing in intensity more rapidly than the violet when the pressure increases.

In absorption, the $(0,0)$ and $(1,0)$ bands of the red system are much weaker than the violet bands at any temperature of the furnace. A list of the stronger absorption lines of the $\lambda 7873$ band is given.

The relative intensities of the two systems in emission in the electric furnace at different temperatures and in the arc are explained by the Boltzmann populations of the upper levels in thermodynamic equilibrium. These populations differ strongly because the upper electronic level, $A^{2} \Pi$, of the red system is much lower (e.p., $1.35 \mathrm{v}$.) than the upper level, $B^{2} \Sigma$, of the violet system (3.2 v.). From the equal intensity of the strongest lines of the two systems in emission at $T=2300^{\circ} \mathrm{C}$, an approximate value of 1400 is found for the ratio of the emission transition probabilities of the strongest violet lines to those of the strongest red lines. The corresponding estimated value of the ratio of the absorption transition probabilities is 87.5, explaining the weakness of the red system in absorption in the laboratory.

These considerations show that the red bands of $C N$ should not be expected in fluorescence in comets and that previous identifications must be revised accordingly. No line of the red system of $C N$ will be found in interstellar absorption. The intensity of the red bands in absorption in certain carbon stars in which the violet bands are weak indicates that the atmospheres of these stars have much less continuous absorption in the red than in the violet.
\end{abstract}

\section{EXPERIMENTAL}

Two groups of cyanogen bands, known as the violet and red systems, are emitted with high intensity in the electric furnace when nitrogen is present, provided the temperature is high enough to give considerable carbon vapor from the material of the graphite tube. The experiments to be described were undertaken for the purpose of observing any changes in relative intensity which might take place at different temperatures. Representative bands of the two systems, although differing greatly in wave length, were photographed in close proximity on the plate by using superposed first and second orders of the 15 -foot concave grating. These are the $(0,0)$ band $\lambda 3883$ and the $(1,0)$ band $\lambda 7873$, degraded toward shorter and longer waves, respectively. The Eastman I-N plate, sensitive to the near infrared and also in moderate degree to the violet, gave the two bands with comparable intensities.

For the earlier experiments, air at various pressures was used in the furnace chamber as commercial tank nitrogen was not available. Later, a tank of pure nitrogen was kindly furnished by the cryogenic laboratory of the California Institute of Technology, and the experiments were in part repeated. The pure nitrogen proved especially effective for absorption spectra; it also eliminated oxidation of the furnace tube.

\section{EMISSION SPECTRUM}

Change in relative intensity with temperature.-Spectrograms were made with the furnace tube at temperatures from $1800^{\circ}$ to $2600^{\circ} \mathrm{C},{ }^{1}$ the pressure being near $1 \mathrm{~cm}$ of mer-

${ }^{1}$ In the description of experiments, centigrade temperatures are given in accordance with the practice in previous papers on electric-furnace spectra. In the discussion the approximate values on the Kelvin scale are used. 
cury and the air or nitrogen being renewed before each exposure. At the lowest temperature, not rising above $1850^{\circ}$ during the rather long run, the strongest lines of the $\lambda 7873$ band (at some distance from the head) appeared faintly, while the head of $\lambda 3883$, much the strongest part of the violet band, did not show. At $1900^{\circ}$, the head and coarse structure of $\lambda 7873$ appeared, and a faint trace of the head of $\lambda 3883$ could be seen. At $2000^{\circ}$, $\lambda 7873$ was still stronger than $\lambda 3883$, but the second head of the latter band at $\lambda 3871$ became visible. Approximate equality of the main structure of the violet and the red bands was attained at $2300^{\circ} ; \lambda 3883$ was somewhat the stronger at $2600^{\circ}$. At $2600^{\circ}$, when the pressure in the furnace was reduced below $1 \mathrm{~mm}$ so that both bands were weak through lack of nitrogen, $\lambda 3883$ was clearly the stronger.

This evidence that $\lambda 7873$ is relatively stronger than $\lambda 3883$ at low temperature is borne out in a striking manner by the intensities of the two bands in the carbon arc, the spectrum of which was photographed on each plate adjacent to the furnace spectra. The temperature of such an arc, burning quietly in air, was determined by Lochte-Holtgreven and Maecker ${ }^{2}$ from the structure of the $\lambda 3883$ band to be $7300^{\circ} \mathrm{C}$. Values by other authors range from $6000^{\circ}$ to $8000^{\circ} \mathrm{C}$. On our arc spectrograms, $\lambda 3883$ is immensely stronger than $\lambda$ 7873, the first ghost of $\lambda 3883$ being comparable with the stronger portions of the $\lambda 7873$ band. By the method of relative exposures for equality of $\lambda 3883$ and its ghost,

TABLE 1

RELATIVE INTENSITIES AT VARIOUS TEMPERATURES

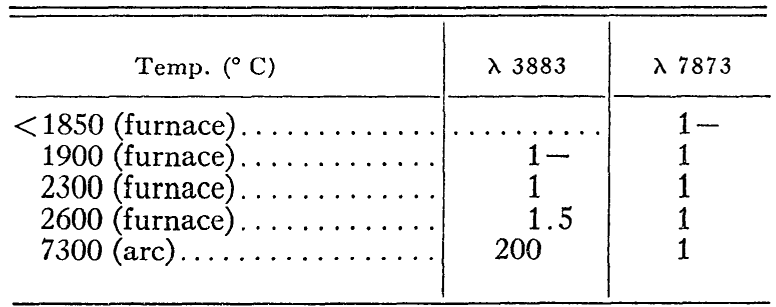

this difference appeared to be of the order of 200 to 1 . Thus the high temperature of the arc gives a ratio of the two bands which is an extension of the intensity change observed in the furnace spectra.

The possibility was considered that the large difference between furnace and arc intensities of $\lambda 3883$ might be due, at least in some degree, to the presence of absorbing vapor, weakening the violet more than the infrared. Opposed to this is the fact that $\lambda 3883$ gains relatively at higher furnace temperatures which should produce more absorbing vapor. A further test was made by placing a carbon arc behind the furnace and focusing its image at the middle of the furnace tube, whence its light passed to the spectrograph. The arc band $\lambda 3883$ was then photographed with the furnace cold and also when heated to $2600^{\circ} \mathrm{C}$. No change appeared in the intensity of the band under the two conditions except that the band head was reversed when the furnace was heated, an effect due to the arc radiation, focused in the tube, acting as a very hot background absorbed by the cooler vapor inside the furnace.

Estimates of the relative intensities of $\lambda 3883$ and $\lambda 7873$ in emission are summarized in Table 1. Most of the energy of the violet system is concentrated in $\lambda 3883$, while only a small fraction of that in the widely distributed red system is in $\lambda 7873$. The relative intensity of the infrared system at low temperature is thus much greater than appears from the visual estimates.

Relative tendency to self-reversal.-Although for temperature comparisons the pressure of nitrogen in the furnace was kept low, the effect of higher pressures was also observed.

${ }^{2}$ Zs.f. Phys., 105, 1, 1937. 
When a current of nitrogen was passed through the furnace tube at atmospheric pressure, $\lambda 3883$ showed partial self-reversal at $2400^{\circ} \mathrm{C}$, the first and second heads being fully reversed at $2600^{\circ}$. Under the same condition, not only the head of $\lambda 7873$ but also the strong lines throughout the band remained sharp. This difference in sensitiveness to absorption agrees with the behavior of the bands in actual absorption spectra, to be described later.

Relative change in intensity with pressure.-The effects of different pressures, from $1 \mathrm{~cm}$ to atmospheric, were observed at a temperature of $2300^{\circ} \mathrm{C}$. With the same exposure times, doubling the pressure gave an approximate doubling of the strength of $\lambda 7873$, while the change in $\lambda 3883$ did not exceed 25 per cent. An uncertainty in this comparison

TABLE 2

WAVE LENGTHS AND ABSORPTION INTENSITIES OF STRONGER LINES OF $(1,0)$ BAND OF RED SYSTEM

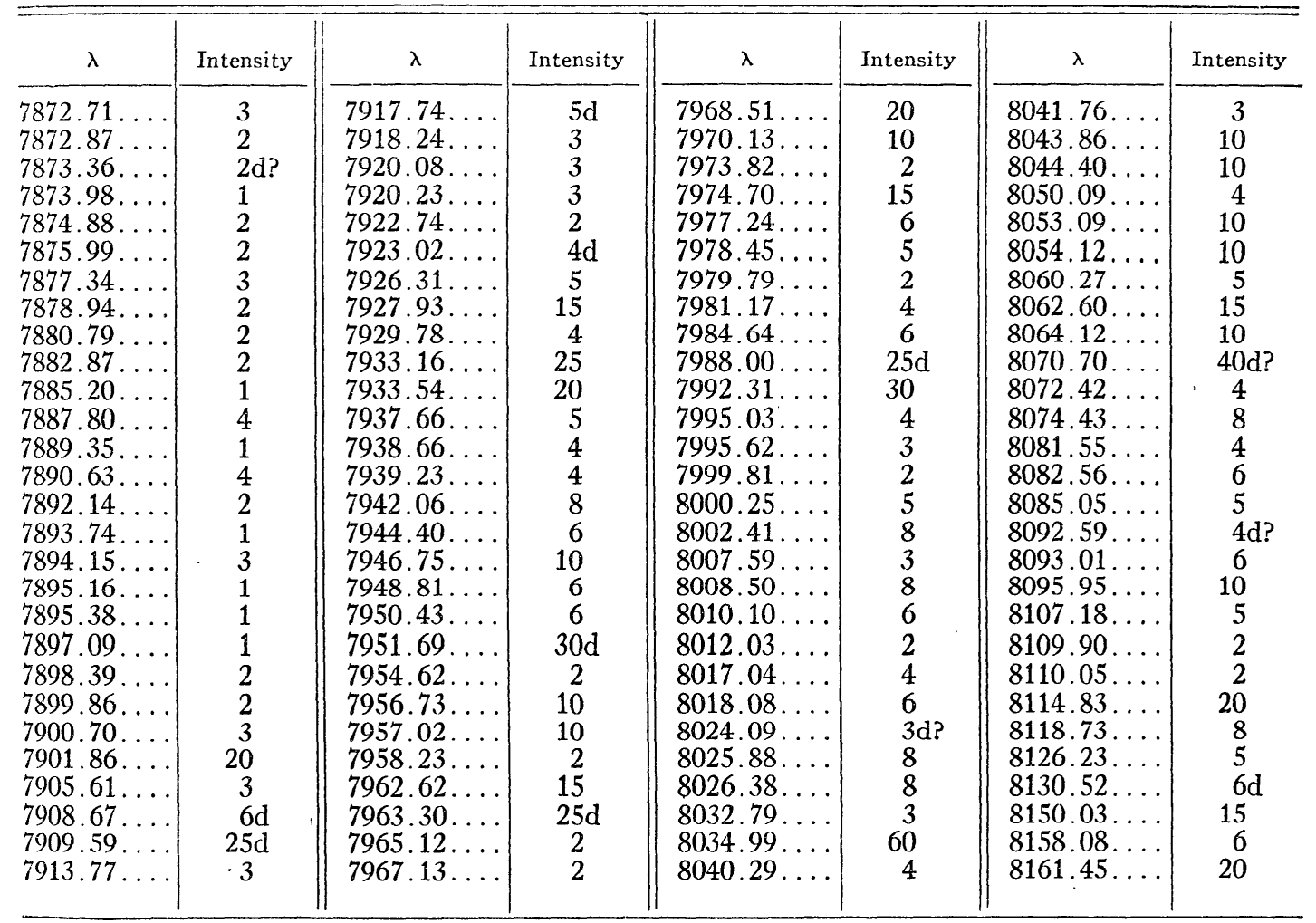

is caused by the easier reversibility of $\lambda 3883$, which at pressures of $40 \mathrm{~cm}$ and higher affects the heads and must produce a softening of the band lines in general. However, the reversal of the heads is not strong at $2300^{\circ}$; and comparison of the bands at lower pressures makes it reasonably certain that the more rapid strengthening of $\lambda 7873$ with increasing pressure is real.

\section{ABSORPTION SPECTRA}

For observing the two band systems in absorption, a continuous background was provided by a tungsten projection lamp with quadruple spiral filament, heated to approximately $3000^{\circ} \mathrm{C}$. A beam concentrated by a lens passed through the furnace tube to the spectrograph slit. As the exposure required to give proper density of the continuous spectrum in the violet was much longer than in the infrared and as no available filter with 
transmission in the violet would fully block out the long waves, an ordinary E 40 plate and a I-N plate were used together to record the two spectral regions. Successive exposures, timed for the two regions, were made with conditions in the furnace unchanged.

With air in the furnace, experiments at different temperatures and for pressures up to atmospheric brought out the full structure of the $\lambda 3883$ band in absorption if the temperature was high enough to produce considerable carbon vapor; but no absorption of $\lambda 7873$ could be seen. The very different behavior of the two $C N$ systems in emission and in absorption is radically at variance with what is commonly found for atoms, for which, if the continuous source is sufficiently hot, the absorption spectrum is very nearly the reversal of the emission spectrum at the same temperature.

As a comparison of corresponding bands in the two systems was desirable, the spectrograph was adjusted to include the $(0,0)$ band $\lambda 9140$ of the red system as well as the $(0,0)$ and $(0,1)$ bands $\lambda 3883$ and $\lambda 4216$ of the violet system, the I-Q plate being used for the entire range. With air in the furnace, $\lambda 9140$ could not be detected with certainty in absorption, although traces of its strongest lines may have been present; $\lambda 3883$ was distinct, as was also the head of $\lambda 4216$, although the violet was deficient in exposure and blurred by overlapping infrared continuous ground. That conditions of the experiment were correct for the production of infrared absorption lines was shown by the strong absorption of the low-temperature pairs of potassium and caesium, near $\lambda 7873$ and $\lambda$ 9140, respectively, when small amounts of the chlorides of these elements were vaporized in the furnace.

Although these experiments with air clearly demonstrated the relative weakness of the red system in absorption, further experiments were made in which a stream of pure nitrogen was passed through the furnace tube at atmospheric pressure. The bands at $\lambda 9140$ and $\lambda 7873$ now appeared faintly in absorption, as did also the $(2,0)$ band at $\lambda 6954$. Their strength was still very low compared with $\lambda \lambda 3590,3883$, and 4216 of the violet system, some or all of which appeared on the same spectrograms.

The new Eastman IV-N plate gave better definition of $\lambda 7873$ in absorption. Since this band is likely to be the strongest of the red system accessible in stellar spectra, and therefore the best for identification of $C N$, a list of the stronger absorption lines with their intensities is given in Table 2. These were selected from the furnace absorption spectrum, but, since few of them were strong enough in absorption to permit satisfactory measures, they were measured in the arc spectrum from iron-arc standards. Wave lengths thus obtained for a number of the best-defined lines were used as standards for check measures on the band lines in the emission-furnace spectrum.

\section{DISCUSSION}

\section{STRUCTURE OF THE RED AND VIOLET SYSTEMS OF $C N$}

Certain important characteristics of the $C N$ bands may now be summarized. The red system is due to a transition from the low electronic state, $A^{2} \Pi_{\text {inv }}$ (excitation potential about $1.35 \mathrm{v}$.), to the ground electronic level, $X^{2} \Sigma^{+}$. Each band is degraded to the red and has four heads $R_{2}, R_{1}, Q_{1}$, and ${ }^{S} R_{21}$. The $(0,0)$ band is at $\lambda 9140\left(R_{2}\right.$ head). On the longwave-length side of $(0,0)$, only the $(1,1)$ transition at $\lambda 9393$ has been photographed. The violet system, which has been studied much more extensively than the red, corresponds to a transition from the higher state $B^{2} \Sigma^{+}\left(\mathrm{EP}\right.$, about $3.2 \mathrm{v}$.) to the ground level $X^{2} \Sigma^{+}$. The strong bands are degraded to the violet.

The two systems have very different intensity distributions both among the vibrational transitions and among the rotational lines of the individual bands. In the violet system only four sequences $\Delta v=$ const. are well marked: $\lambda 3883(\Delta v=0), \lambda 3590(\Delta v=+1)$, $\lambda 4216(\Delta v=-1)$, and $\lambda 4606(\Delta v=-2)$, the $(0,0)$ transition giving by far the strongest band. The intensity distribution among the vibrational transitions is represented by a very narrow Franck-Condon parabola. In the red system the Franck-Condon parabola 
is wider, and many more vibrational transitions are present than in the violet system. In fact, since the red system, with its $(0,0)$ band at $\lambda 9140$, lies at the end of the ordinary photographic range, only the short-wave-length half of the spectrum has been observed. Even this half, however, extends over a wave-number range of $12,200 \mathrm{~cm}^{-1}$, while the observed violet system covers only $7000 \mathrm{~cm}^{-1}$. These features explain why the red system appears of much more uniform intensity than the violet system. The contrast is enhanced by the difference in distribution of rotational intensity. The value of $r_{e}^{\prime}-r_{e}^{\prime \prime}$ for the violet system is only $-0.021 \mathrm{~A}$, while for the red system it is $+0.067 \mathrm{~A}$. Hence, in the red bands the heads are nearer the origins than in the violet bands, and at the temperatures of the furnace or the arc the red bands will not show the dense crowding of lines at the heads characteristic of the violet bands.

\section{RELATIVE BEHAVIOR OF THE TWO SYSTEMS IN EMISSION}

The excitation energies $A_{0}$ and the vibrational frequencies $\omega_{e}$ of the $A^{2} \Pi$ and $B^{2} \Sigma$ terms are:

$$
\begin{array}{lll}
\text { For } A^{2} \Pi, & A_{0}=10929.3 \text { and } 10877.1 \mathrm{~cm}^{-1} ; & \omega_{e}=1788.7 \mathrm{~cm}^{-1} ; \\
\text { For } B^{2} \Sigma^{+}, & A_{0}=25797.8 \mathrm{~cm}^{-1} ; & \omega_{e}=2164.1 \mathrm{~cm}^{-1} .
\end{array}
$$

In thermodynamic equilibrium, the populations on the levels $A^{2} \Pi, v^{\prime}=1$, and $B^{2} \Sigma, v^{\prime}=$ 0 , are in the ratio given by the Boltzmann relation. The intensity ratio of the $\lambda \mathbf{3 8 8 3}$ and $\lambda 7873$ emission bands at $T=2000^{\circ} \mathrm{K}$ may be designated by $a$; at higher temperatures the intensity ratio increases rapidly, reaching the value $848 a$ at $7000^{\circ} \mathrm{K}$ (Table 3 ). Evi-

TABLE 3

INTENSITY RATIO OF THE VIOLET BAND $\lambda 3883$ AND OF THE RED

\begin{tabular}{|c|c|c|c|}
\hline$\left(\right.$ in $\left.^{\circ} \mathrm{T}\right)$ & $\begin{array}{l}\text { Int. Ratio } \\
3883 / 7874\end{array}$ & $\left(\right.$ in $\left.^{\circ} \mathrm{K}\right)$ & $\begin{array}{l}\text { Int. Ratio } \\
3883 / 7874\end{array}$ \\
\hline $\begin{array}{l}2000 \ldots \ldots \ldots \ldots \ldots \ldots \\
2300 \ldots \ldots \ldots \ldots \ldots \ldots \\
2600 \ldots \ldots \ldots \ldots \ldots \ldots \\
3000 \ldots \ldots \ldots \ldots \ldots \ldots\end{array}$ & $\begin{aligned} & a \\
& 3.425 a \\
& 8.834 \\
& 23.265 \\
& 111.5\end{aligned}$ & $\begin{array}{l}5000 \ldots \ldots \ldots \ldots \ldots \ldots \\
6000 \ldots \ldots \ldots \ldots \ldots \ldots \ldots \\
7000 \ldots \ldots \ldots \ldots \ldots \ldots \\
8000 \ldots \ldots \ldots \ldots\end{array}$ & $\begin{array}{l}288.7 a \\
541 \\
848 \\
1188\end{array}$ \\
\hline
\end{tabular}
BAND $\lambda 7874$ AT VARIOUS TEMPERATURES

dently, thermodynamic equilibrium is only an approximation, but this approximation is, in fact, close and suffices to explain the sharp increase of the violet system with respect to the red one with increasing temperature. The theoretical factor of increase in intensity ratio from $T=2600^{\circ} \mathrm{K}$ to $T=7600^{\circ} \mathrm{K}$ is 120 , as against the estimated experimental value of 200 . The figure 200 , however, would be the theoretical factor of increase from $T=2430^{\circ} \mathrm{K}$ (or $2160^{\circ} \mathrm{C}$ ) to $T=7600^{\circ} \mathrm{K}$. The agreement between the experimental and the theoretical values is thus quite satisfactory.

In computing Table 3, account has been taken of the vibrational energy of the level $v^{\prime}=1$ of $A^{2} \Pi$, from which the $\lambda 7873$ band arises. The ratios would be higher for the $(0,0)$ band $\lambda 9140$. If we designate by $\beta$ the intensity ratio of the $(1,0) \lambda 7873$ and the $(0,0) \lambda 9140$ bands at $T=2000^{\circ} \mathrm{K}$, the values at higher temperatures may again be obtained by the Boltzmann formula. They are listed in Table 4, which shows, for example, that the intensity ratio of the $\lambda 3883$ and $\lambda 9140$ bands increases by a factor $848 \times 2.47$ $=2095$ from $2000^{\circ} \mathrm{K}$ to $7000^{\circ} \mathrm{K}$.

The more rapid intensity strengthening with pressure of $\lambda 7873$ compared with $\lambda 3883$ 
is probably an effect of the more pronounced collisional de-excitation on the high level $B^{2} \Sigma$ compared with the low level $A^{2} \Pi$. Similar greater de-excitation on more highly excited levels has been studied in fluorescence phenomena.

At $2600^{\circ} \mathrm{K}$ the relative Boltzmann populations on the levels $A^{2} \Pi_{3 / 2}, v^{\prime}=1$, and $B^{2} \Sigma_{1 / 2}$, $v^{\prime}=0$, are 2850:1, account being taken of the statistical weights. The intensities of characteristic lines of the $\lambda 3883$ and $\lambda 7873$ bands being approximately equal at that temperature, the ratio of the emission transition probabilities, ${ }^{3} A_{v}$ and $A_{r}$, of the lines considered in the violet and red bands is approximately 2850 times the ratio of the wave numbers of the red and violet lines. The transition probability, $A_{v}$, of the violet lines is thus approximately 1400 times the transition probability of the red lines.

This value 1400 may be in error by a factor possibly as high as 3 , since no account has been taken of collisional de-excitation, reabsorption, and other sources of error. Moreover, since the equality of emission intensity is a simple visual estimate on spectrograms, there

TABLE 4

INTENSITY RATIO OF THE $(1,0) \lambda 7873$ AND THE $(0,0) \lambda 9140$

BANDS AT VARIOUS TEMPERATURES

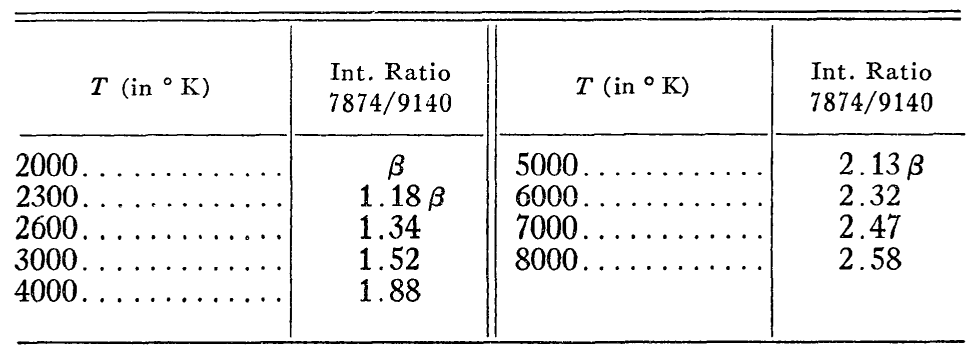

may be some error in the determination of the temperature at which the actual equality is reached. But it is, at any rate, certain that the violet emission bands have a much higher transition probability than the red bands.

\section{RELATIVE BEHAVIOR OF THE TWO SYSTEMS IN ABSORPTION}

From the results on the emission transition probabilities we understand readily that the violet system should appear much more strongly in absorption than the red system. In fact, we at once obtain estimates of the ratios of the absorption transition probabilities $B_{v}$ and $B_{r}$ and of the oscillator strengths $f_{v}$ and $f_{r}$ from the estimated ratio $A_{v} / A_{r}$ as follows:

$$
\begin{gathered}
\frac{B_{v}}{B_{r}}=\frac{A_{v}}{A_{r}} \times \frac{g\left({ }^{2} \Sigma\right)}{g\left({ }^{2} \Pi\right)} \times \frac{\nu_{r}^{3}}{\nu_{v}^{3}}=1400 \times \frac{2}{4} \times\left(\frac{1}{2}\right)^{3}=87.5, \\
\frac{f_{v}}{f_{r}}=\frac{\nu_{v}}{\nu_{r}} \times \frac{B_{v}}{B_{r}}=2 \times 87.5=175 .
\end{gathered}
$$

With the furnace at $T=2650^{\circ} \mathrm{K}$, the strongest line in the $\lambda 7873$ band is much weaker than the line $\mathrm{P}(1)$ in the $(0,0)$ violet band; the ratio appears visually of the order of 10. Theoretically, the $\mathrm{P}(1)$ line itself is approximately one-twelfth as strong as the strongest individual features of the $\lambda 3883$ band. Hence for the ratio $B_{v} / B_{r}$ we can readily accept the value 87 obtained from $A_{v} / A_{r}$. A direct determination of $B_{v} / B_{r}$ would require photometric measurements on the absorption spectra, which cannot be carried out at present.

${ }^{3}$ The subscripts $v$ and $r$ as used with the letters $A, B, f$, and $\nu$ refer, respectively, to the violet and red systems (not to vibration and rotation!). 
In comparing absorption bands of $C N$ at different temperatures, account must evidently be taken of the population on the lower vibrational level. For example, if we designate $\gamma$ as the intensity ratio of the red absorption bands $\lambda 6355(4,1)$ and $\lambda 7873(1,0)$ at $T=4300^{\circ} \mathrm{K}$, the ratio would become $0.88 \gamma$ at $3650^{\circ} \mathrm{K}$ and $0.74 \gamma$ at $3000^{\circ} \mathrm{K}$.

It should be emphasized that the values obtained for the ratios of the transition probabilities $A_{v} / A_{r}$ and $B_{v} / B_{r}$ apply to the individual lines considered on the grating spectrograms. These ratios corresponding to individual lines are the main factors in which astronomers are interested. But the value obtained for $A_{v} / A_{r}$ should not be construed as meaning that the $B^{2} \Sigma \rightarrow X^{2} \Sigma$ electronic transition has an emission probability 1400 times larger than the $A^{2} \Pi \rightarrow X^{2} \Sigma$ transition. To obtain the value of the total electronic transition probabilities, account should be taken of the intensity distributions of the individual lines within the bands, and of the bands within the systems. These rotational and vibrational factors are very different for the two systems, but a numerical estimate of the factors would require a considerable amount of calculation. A total factor of at least 10 may be expected, ${ }^{4}$ reducing the ratio of the electronic emission transition probabilities to a value much smaller than 1400 .

Evidently the high transition probability of the violet bands compared with the red bands explains the difference in self-reversal, which occurs easily in the violet system but not in the red.

\section{ASTROPHYSICAI APPLICATIONS}

COMETARY SPECTRA

The intensity of the violet bands in emission in all comets has suggested to many observers that a number of the emission features in the visual region were due to the red

TABLE 5

Emission Features in Various Comets Attributed to the Red System of $C N$

\begin{tabular}{|c|c|c|c|c|c|c|c|c|c|}
\hline \multirow{3}{*}{$\begin{array}{c}\text { AverAGE COME- } \\
\text { TARY } \lambda \text { AND } \\
\text { INTENSITY }\end{array}$} & \multicolumn{3}{|c|}{$\begin{array}{l}\text { IdENTIFICATIONS PREVI- } \\
\text { ousLy SUGGested with } \\
\text { RED } C N \text { Bands }\end{array}$} & \multirow{3}{*}{$\begin{array}{c}\text { MORE } \\
\text { PROBABLE } \\
\text { IDENTIFI- } \\
\text { CATION }\end{array}$} & \multirow{3}{*}{$\begin{array}{l}\text { AvERAGE COME- } \\
\text { TARY } \lambda \text { AND } \\
\text { INTENSITY }\end{array}$} & \multicolumn{3}{|c|}{$\begin{array}{l}\text { IDENTifications Previ- } \\
\text { ously SUGGested with } \\
\text { RED CN BANDS }\end{array}$} & \multirow{3}{*}{$\begin{array}{c}\text { MORE } \\
\text { PROBABLE } \\
\text { IDENTIFI- } \\
\text { CATION }\end{array}$} \\
\hline & \multirow{2}{*}{$\begin{array}{c}\text { Transi- } \\
\text { tion }\end{array}$} & \multicolumn{2}{|c|}{ Lab. Intensity } & & & \multirow{2}{*}{$\begin{array}{c}\text { Transi- } \\
\text { tion }\end{array}$} & \multicolumn{2}{|c|}{ Lab. Intensity } & \\
\hline & & Flame & $\begin{array}{c}\text { Dis- } \\
\text { charge }\end{array}$ & & & & Flame & $\begin{array}{c}\text { Dis- } \\
\text { charge }\end{array}$ & \\
\hline $4839(1-2)$ & $(7,1)$ & 1 & $?$ & & $6200(0+)$ & $(3,0)$ & 6 & 4 & \\
\hline $4937(1-0)\}$ & $(8,2)$ & 1 & 2 & & $\left.\begin{array}{l}6279(0) \\
6289(0)\end{array}\right\}$. & $(9,5)$ & & 1 & $\mathrm{NH}_{2}$ \\
\hline $\begin{array}{l}4953(1-0) \\
5243(1) \ldots\end{array}$ & $(6,1)$ & 2 & 3 & $\mathrm{NH}_{2}$ & $6333(2)$. & $(4,1)$ & 10 & 9 & $\mathrm{NH}_{2}$ \\
\hline $5355(1-2)$ & $(7,2)$ & 2 & 4 & & $6434(0)$. & $(10,6)$ & & 1 & $\mathrm{NH}_{2}$ \\
\hline $5740(1-2)\}$ & $(5,1)$ & 5 & 7 & $\mathrm{NH}_{2}$ & $6474(0)$ & $(5,2)$ & 9 & 10 & $\begin{array}{l}\mathrm{NH}_{2} ? \\
\mathrm{NH}_{2} ?\end{array}$ \\
\hline $5800(0-1)$. & $(6,2)$ & 2 & 9 & $\mathrm{NH}_{2}$ ? & $6642(1) \ldots$ & $(6,3)$ & & & $\mathrm{NH}_{2}$ ? \\
\hline $5996(2) \ldots$ & $(7,3)$ & 2 & 6 & $\mathrm{NH}_{2}$ & & & & & \\
\hline
\end{tabular}

system. This suggestion has been made for Comets Brooks, Halley, Peltier, Finsler, Cunningham, and Whipple II. The proposed identifications were not convincing to most ob-

\footnotetext{
${ }^{4}$ This crude estimate is obtained by noting that the $(0,0)$ or $(1,0)$ red band does not contain more than 20 per cent of the total intensity of all the red absorption bands with lower-level $v^{\prime \prime}=0$, while the $(0,0)$ violet band takes most of the total intensity of all the violet transitions from $v^{\prime \prime}=0$. Moreover, there are eight branches in each red band instead of two in a violet band.
} 
servers, since the relative intensities of the cometary bands differed greatly from the laboratory intensities, sometimes even in bands having the same upper vibrational level. From the numerous red $C N$ bands and the various features of cometary spectra in the red, we should expect a number of accidental coincidences in observed wave length, especially with low dispersion. The situation remained doubtful until quite recently.

Numerous investigations published in recent years have definitely shown that the bands of a comet are emitted by pure resonance fluorescence excited by solar radiation. The fluorescence hypothesis can thus be applied to the red bands without hesitation. To be emitted, the red bands require the absorption of solar radiation by the $C N$ molecules of the comet. Because of the very small absorption transition probability of the red bands relative to the violet, the red bands should be so extremely weak in fluorescence emission compared to the violet bands that their observation would be impossible. For this reason identifications of the red $C N$ bands should find a place in future descriptions of cometary spectra only after much more careful discussion than heretofore.

We have compiled in Table 5 the cometary emissions that have been occasionally attributed to the red system of $C N$. These lines do not all appear in any specific object but are listed together for the sake of simplicity. ${ }^{5}$ Even from Table 5 the anomalous intensities are apparent (compare, for example, the intensities of the 5,1 and 5,2 bands). The anomalies are, however, still more striking when the whole table of laboratory bands is considered, since many transitions that are strong in the laboratory are absent in comets. Most of the emissions of Table 5 are actually due to $\mathrm{NH}_{2}{ }^{6}$

\section{INTERSTELLAR ABSORPTION}

W. S. Adams ${ }^{7}$ did not find any trace of interstellar lines belonging to the red $C N$ system, in the region $\lambda \lambda$ 6500-9000 of the star $\zeta$ Ophiuchi, which shows relatively strong interstellar $C N$ lines in the region of $\lambda$ 3880. The interstellar $K$ I line at $\lambda 7699$ is well seen on the infrared spectrograms. The absence of interstellar red $C N$ lines is readily understood because of the small value of the transition probability.

\section{CARBON STARS}

The infrared $C N$ bands have been observed by P. W. Merrill, ${ }^{8}$ R. F. Sanford, ${ }^{9}$ and others in the carbon stars where they reach a high intensity. Cyanogen bands in the visual region have also been frequently observed. In certain carbon stars, such as $U$ Hydrae (class N2 or C7), the infrared lines of $C N$ are of considerable strength, while the violet $(0,1)$ band at $\lambda 4216$ is very weak or absent, although the continuous background near by is still strong enough to reveal absorption features. ${ }^{10}$ From the high ratio of the absorption transition probabilities of the violet and red systems one may conclude that the peculiar behavior of the $C N$ bands in $\mathrm{N}$ stars is due to a large variation in general opacity of the stellar atmosphere between the red and the violet regions. The continuous absorption of the atmosphere in the red must be very small compared to that in the violet. In other carbon stars (class $\mathrm{R}$ or early $\mathrm{C}$ ), red $C N$ bands are observed, together with strong bands of the $\Delta v=-2,-1,0,+1$, and +2 sequences; for these stars the variation of atmuspheric opacity with wave length thus differs from that in U Hydrae. As a

${ }^{5}$ References for the individual objects are: Comet Brooks, W. H. Wright, Lick Obs. Bull., 7, 8, 1912; Comet Halley, N. T. Bobrovnikoff, Lick Obs. Pub., 17, 435, 1931; Comet Peltier, W. Baade and R. Minkowski, Pub. A.S.P., 48, 277, 1936; Comet Finsler, R. Minkowski, Pub. A.S.P., 49, 276, 1937; Comets Cunningham and Whipple II, P. Swings, A. McKellar, and R. Minkowski, Ap. J., 98, 142, 1943.

${ }^{6}$ Swings, McKellar, and Minkowski, loc. cit.

7 Annual Report of the Mount Wilson Observatory for 1942-1943, p. 15.

${ }^{8}$ Mt.W. Contr., No. 486; Ap. J., 79, 183, 1933.

${ }^{9}$ Mt. W. Contr., No. 525; Ap. J., 82, 202, 1935.

${ }^{10}$ Dr. R. F. Sanford, private communication. 
rule the violet bands decrease steadily in intensity from $\mathrm{C} 4$ to $\mathrm{C} 9$, while the red bands remain more or less constant. ${ }^{11}$ This would mean that the continuous absorption in the violet increases steadily from $\mathrm{C} 4$ to $\mathrm{C} 9$ relative to its value in the red.

The continuous absorption causing the weakening of the bands in the violet region of certain carbon stars is related to the steep gradients in the continuous spectrum found for the same stars in the short-wave-length region. ${ }^{11}$ It explains also why different $C N$ bands, appearing in different spectral regions, may reach their intensity maximum at different spectral types. In any discussion of the intensity behavior of molecular bands, stellar opacity should be considered as well as molecular abundance and population on the vibrational level corresponding to the band. To certain writers the behavior of $C N$ bands originating from the same lower electronic level appeared so anomalous that the observational data were suspected of being erroneous. ${ }^{12}$

Needless to say, the dispersion used in the stellar spectrograms affects the relative ease of detection of the red and blue bands on account of the very different intensity distribution among the rotational lines.

Our thanks are due to Dr. H. N. Russell, Dr. R. S. Mulliken, and Dr. R. T. Birge for valuable suggestions.

${ }^{11}$ P. C. Keenan and W. W. Morgan, Ap. J., 94, 501, 1941.

${ }^{12}$ See, e.g., K. Wurm, Zs.f. Ap., 5, 260, 1932. 